\title{
An improved algorithm for step counting based on Android phones Zhang Yong jun ${ }^{\mathrm{a}}$, Zhao Shuaib \\ BUPT, No.10 Xitucheng Road, Haidian District, Beijing \\ ayizhang@bupt.edu.cn, bzhaoshuai_bupt@163.com
}

\section{Keywords: Acceleration Sensor; Smart Phone; Peak Detection; Zero Crossing Detection; Dynamic Threshold}

\begin{abstract}
This paper designs a method based on the combination of zero crossing detection and peak detection algorithm. The main idea of this algorithm is to detect the uplink zero point, the downlink zero point and the peak of the acceleration waveform, and count user's step number by the dynamic threshold of the peak and time interval. In the experiment, we allow users to hold the phone in hand or keep it in the pocket. The validity of the algorithm is verified by experiments.
\end{abstract}

\section{Introduction}

By now the smart phone, especially the Android smart phone is more powerful than before. And more and more people use it in their daily life. With the continuous development of hardware, the smart phone integrates with high-accuracy sensor laid the foundation for many useful applications [1].Step counting is one of useful applications, which has important reference for monitoring the user's daily behavior and health. There exists a few research work and many commercial smart phone applications to detect or count the user's steps. In other work, researchers have tried to detect or count the steps by attaching the phone into a specific position. In this paper, an improved algorithm for step counting based on smart phone is proposed, which allow users to hold the phone in hand or keep it in the pocket.

\section{Existing Algorithms and Problem Analysis}

The waveform of the accelerometer displays some regularity when the samrt phone is carried by pedestrian. Acceleration data is analyzed to detect the number of steps, the position between smart phone and human body is not fixed.The3-axis total acceleration for analysis is a universal method.

The walking acceleration signal shows below as Fig 1:

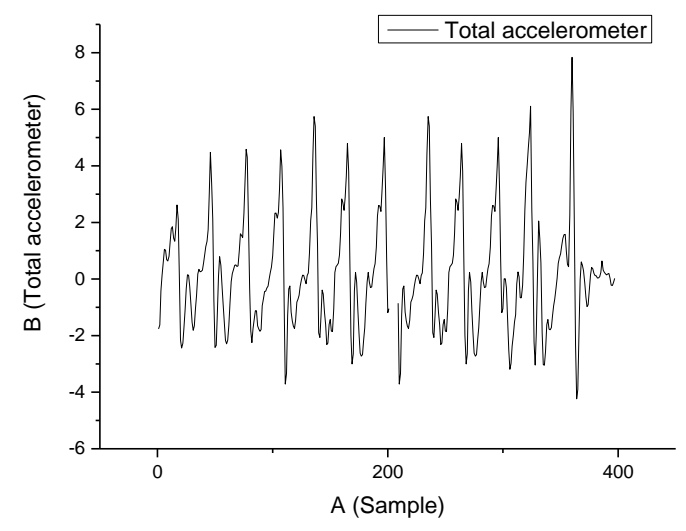

Fig. 1 Acceleration waveform

However, the samrt phone is placed in different positions of the human body and its waveform and cycle are obviously different. There are a large number of algorithms for calculating the acceleration waveform of human body in different position. 
Fast Fourier transform method: Walking at a frequency of about $1 \sim 3 \mathrm{~Hz}$, can be obtained by the peak frequency in the frequency domain.

Zero crossing method: In the process of walking, the normal gait includes lifting foot, swing and landing. Excluding the acceleration of gravity, the total acceleration value will increase when lifting foot and will decrease when landing [2].Thus, the total acceleration value will pass through zero two times.

Peak detection method: In this method, the peak detection is realized by adaptive acceleration threshold analysis, which is mainly aimed at each step of the upper body of the pedestrian [3].

Although there are many kinds of algorithms [4], it can't meet the needs of different applications. The main shortcomings are the following :(1) attaching the phone into a specific position, which is not universal(2) Does not have the characteristics of real-time counting. But taking into account of the position and pose of mobile phone of arbitrary and need to design a universal meter step algorithm [5]. Therefore, in order to overcome the shortcomings of existing algorithms, this paper presents a combination of zero detection and peak detection algorithm.

\section{The main idea of the algorithm}

When walking, it can be divided into the following scenarios: (1) walking in normal speed holding mobile in hand;(2) walking in fast speed holding mobile in hand;(3) walking in slow speed holding mobile in hand;(4)walking in normal speed putting the smart phone in their pocket.

No matter what kind of scene out of the above, the acceleration wave shows regularity. Analysis of a gait waveform, the waveform acceleration values from small to large across zero point is called the uplink zero point, the waveform acceleration values from small to large across zero point is called the downlink zero point.

A gait waveform has the following characteristics, show as Fig.2: (1) A gait waveform has a peak (2) The wave has an uplink zero point (represented by A), a downlink zero point (represented by B).

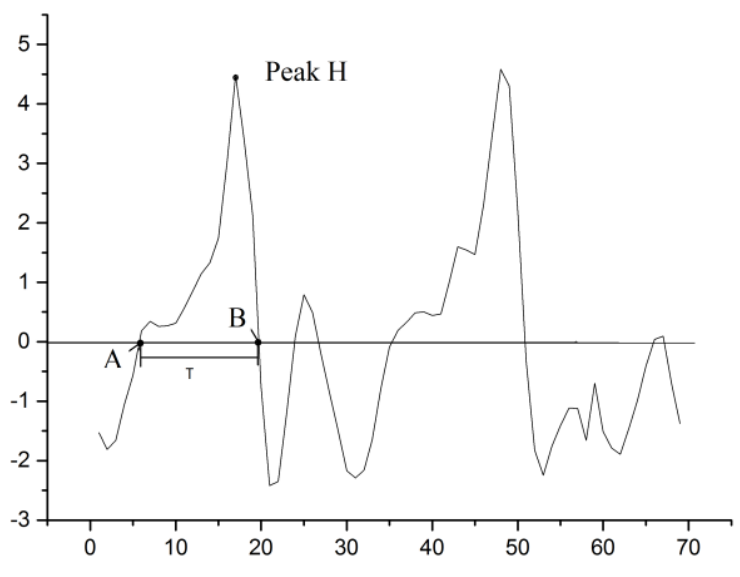

Fig. 2 The wave of gait

\section{The key Algorithm}

Step 1: Get the three axis acceleration (presented by $\mathrm{x}, \mathrm{y}, \mathrm{z}$ respectively) of the smart phone's acceleration sensor at the frequency of $50 \mathrm{~Hz}[6,7]$.Then, calculate the total acceleration minus acceleration of gravity $g, g$ can be tested in the local area by calculating the mean value of five point when the smart is static, which is easy to obtain . The point value $r$ is show as below equation:

$$
\mathrm{r}=\sqrt{x^{2}+y^{2}+z^{2}}-\mathrm{g}
$$

Step 2: If the point value $r$ equal to zero or its previous point multiply the after one less than zero, record the corresponding time of uplink and downlink zero point, presented by $t 1$ and $t 2$ respectively. 
Calculate the time difference $\mathrm{T}$ between the two zeros point. Detect the peak between the uplink and downlink. The value of peak point is $\mathrm{H}$.

Step 3: The initial value of $T t$ and $H t$ is empiric value. If $\mathrm{T}$ is greater than the initial time value $T t$, $\mathrm{H}$ is greater than the initial threshold $H t$, the number of steps plus 1 . While $\mathrm{T}$ and $\mathrm{H}$ will be recorded for the gradient threshold processing updates time and peak value.

Fig. 3 gives the algorithm flowchart.

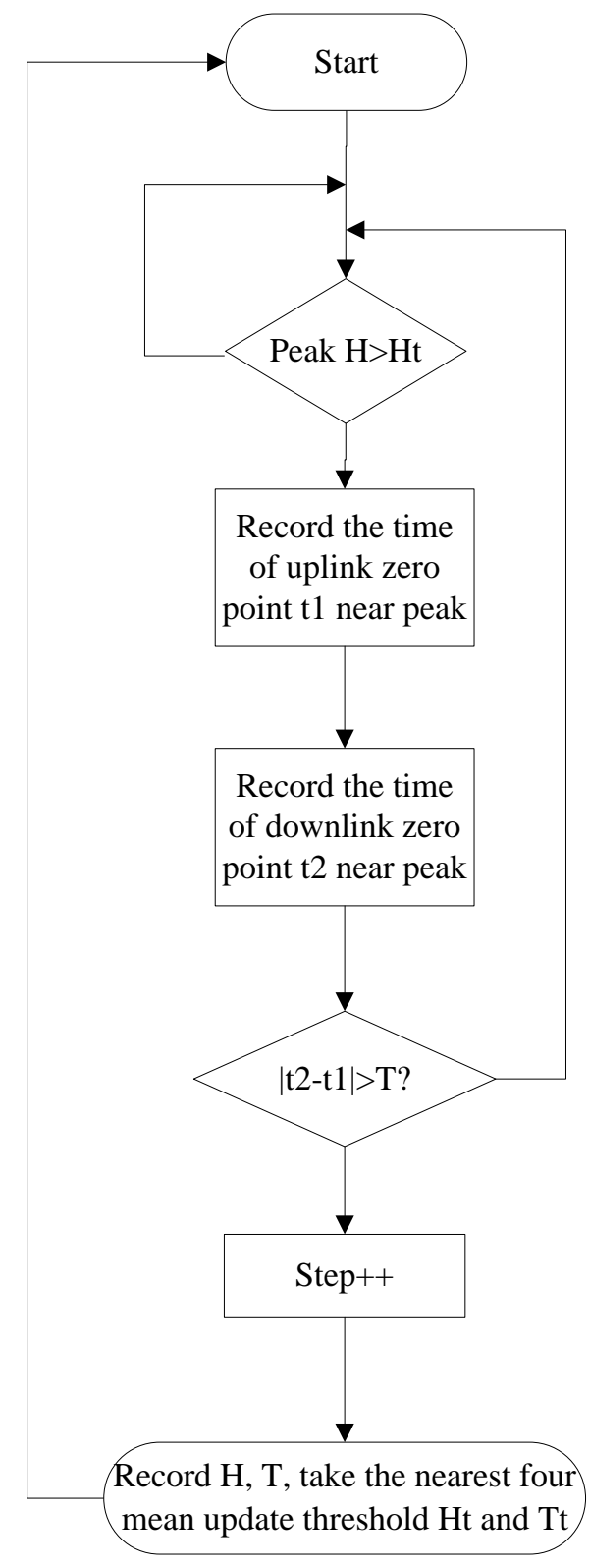

Fig. 3 Algorithm flow chart

\section{Experimental results and analysis}

In the paper, we test the algorithm using HUAWEI glory $4 \mathrm{X}$, the phone system for Android4.4. Test methods for different test using software respectively in 4 kinds of scene walk 100 steps, and statistics for different users of the same scene step counting the accuracy of the results. Contrast the algorithm in the paper, peak detection algorithm and zero crossing algorithms in for walking step counting accuracy. As can be seen from Fig. 4, the accuracy of the proposed algorithm is 96\%-99\%. 


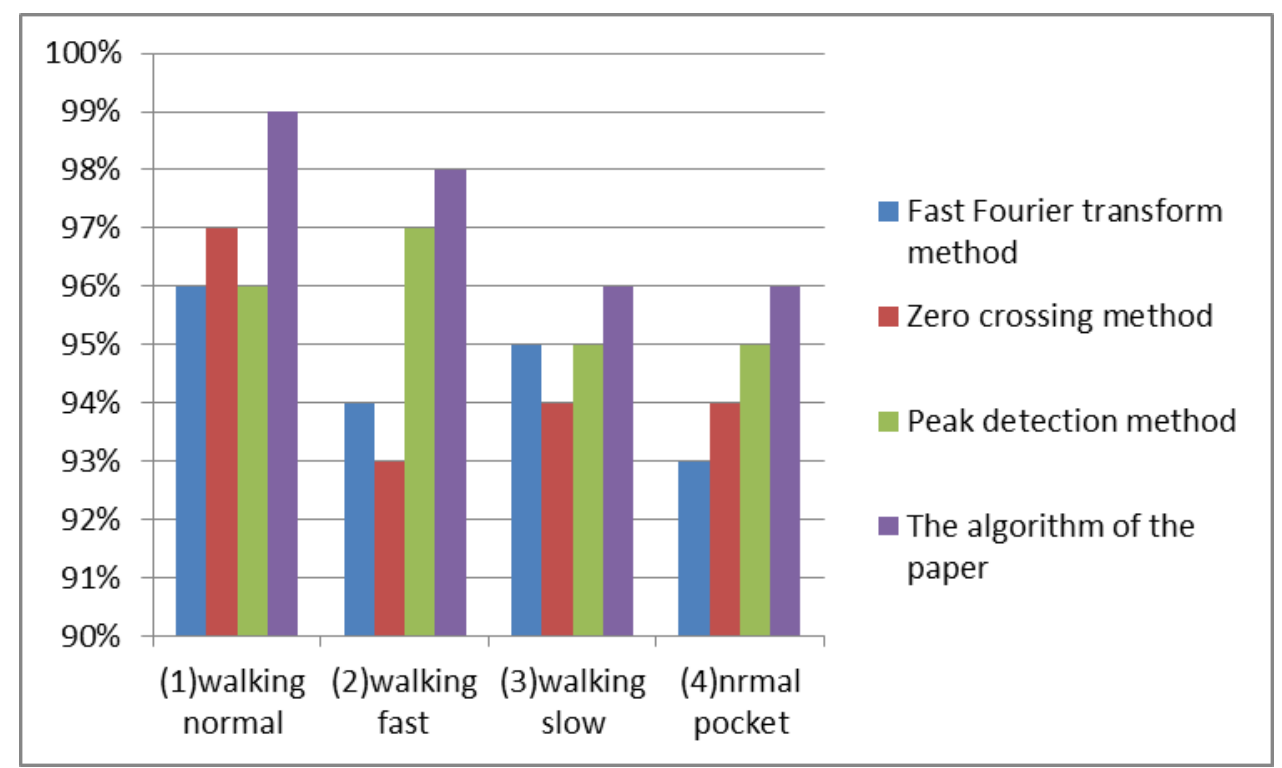

Fig. 4 Comparison of algorithms in different scenarios

\section{Summary}

In this paper, the algorithm based on the acceleration sensor of the mobile phone is improved. The algorithm is universal, not affected by relative position between smart phone and human. The significant contribution in our work are the combination of zero crossing method and peck detection method, the training threshold of time and peak, the counting method.

Finally, the algorithm is applied to the actual inertial navigation system, which proves the feasibility of the algorithm.

\section{References}

[1]Information on http://www.android-doc.com/

[2]Weimann F, Abwerzger G, Hofman-Wellenhof B. 2007. A pedestrian navigation system for urban and indoor environments[C]. Proc. ION GNSS 2007, Fort Worth, TX, USA, 1380-1389

[3]Levi R, Judd T.Dead reckoning navigational system using accelerometer to measure foot impacts [P].U.S.Patent US5583776, 1999.

[4]Lachapelle G, Godha S, Cannon ME.Performance of integrated HSGPS-IMU technology for pedestrian navigation under signal masking[C].Proc.European Navigation Conference, Manchester, $\mathrm{UK}, 1-24$.

[5]Zhao N.Full-featured pedometer design realized with 3-axis digital accederometer[J].Analog Dialogue,2011,6:1-5

[6]YANG M J,ZHENG H R,WANG H Y,et al.Assessing the utility of smart mobile phone in gait pattern analysis[J].Health and Technology,2012,2(1):81-88

[7]PAN M S,LIN H W.A step counting algorithm for smartphone users:Design and implementation[J].Sensors Journal,2015,15(4):2296-2305 